\title{
Escitalopram versus paroxetine controlled release in major depressive disorder: a randomized trial
}

This article was published in the following Dove Press journal:

Neuropsychiatric Disease and Treatment

6 January 2017

Number of times this article has been viewed

\author{
Taro Kishi' \\ Yuki Matsuda' \\ Shinji Matsunaga' \\ Masatsugu Moriwaki ${ }^{1,2}$ \\ Yoichiro Otake ${ }^{2}$ \\ Kaku Akamatsu ${ }^{3}$ \\ Tomo Okochi ${ }^{4}$ \\ Shigeki Hirano ${ }^{4}$ \\ Toshihiko Funahashi ${ }^{5}$ \\ Momoko Okuda ${ }^{6}$ \\ Hideaki Tabuse ${ }^{6}$ \\ Kiyoshi Fujita ${ }^{2}$ \\ Nakao Iwata' \\ 'Department of Psychiatry, \\ Fujita Health University School \\ of Medicine, ${ }^{2}$ Department of \\ Psychiatry, Okehazama Hospital, \\ Toyoake, ${ }^{3}$ Department of Psychiatry, \\ Jindai Clinic, Nagoya, ${ }^{4}$ Department of \\ Psychiatry, Toyota Memorial Hospital, \\ ${ }^{5}$ Department of Psychiatry, Jinda \\ Hospital, Toyota, Aichi, ${ }^{6}$ Department \\ of Psychiatry, Holy Cross Hospital, \\ Toki, Gifu, Japan
}

Correspondence: Taro Kishi Department of Psychiatry, Fujita Health University School of Medicine, Toyoake, Aichi 470-I I 92, Japan

Tel +8I 562939250

Fax+8| 56293 |83।

Email tarok@fujita-hu.ac.jp
Objective: There are no direct comparisons between escitalopram and paroxetine controlled release in patients with major depressive disorder (MDD).

Methods: We conducted a 24-week, rater-masked, randomized trial of escitalopram (5-20 mg/day) versus paroxetine controlled release $(12.5-50 \mathrm{mg} /$ day) in patients with MDD (UMIN000011191). Patients with the diagnosis of moderate-to-severe MDD (a 17-item Hamilton Rating Scale for Depression [HAMD-17], with total score at baseline being $\geq 20$ ) were recruited to participate in a parallel, randomized, controlled trial. The primary outcome for efficacy was an improvement in the 21-item HAMD (HAMD-21) total score at 24 weeks. The secondary outcomes were the response, remission, and discontinuation rates and the incidence of individual adverse events.

Results: A total of 88 patients with MDD (males, 61.4\%; mean age, $40.8 \pm 13.4$ years) were recruited. The discontinuation rate was $58.0 \%$ (escitalopram, 55.8\%; paroxetine controlled release, $60.0 \%$ ). Both escitalopram and paroxetine controlled-release treatment groups exhibited significant reduction in the HAMD-21 total score at 2, 4, 8, 12, and 24 weeks from the baseline. However, there were no significant differences in the HAMD-21 total score, response rate, remission rate, and discontinuation rate at any time point between the groups. In addition, there were no significant differences in the incidence of any individual adverse events (eg, nausea, vomiting, and somnolence) between the treatment groups.

Conclusion: Our results suggest that escitalopram and paroxetine controlled release had similar efficacy and safety profiles in patients with MDD. One of the primary limitations of this study is the small sample size.

Keywords: escitalopram, paroxetine controlled release, major depressive disorder, Hamilton Rating Scale for Depression, antidepressant

\section{Introduction}

In 2009, the Meta-Analysis of New Generation Antidepressants Study2 reported that clinically important differences for both efficacy and acceptability exist among commonly prescribed antidepressants in favor of escitalopram and sertraline. In 2012, escitalopram was demonstrated to have the highest probability of remission and is the most effective and cost-effective pharmacological treatment in a primary care setting. ${ }^{3}$ Escitalopram appears to be the best first-line antidepressant for treating major depressive disorder (MDD). In contrast, in 2010, paroxetine immediate release was the best-selling antidepressant in Japan. ${ }^{4}$

There were three randomized trials of escitalopram versus paroxetine immediate release in patients with MDD. Boulenger et al's study and Kasper et al's study reported that escitalopram is more effective and safer than paroxetine immediate release in the long-term treatment of patients with MDD. ${ }^{5,6}$ Baldwin et al reported that significantly 
$(P<0.01)$ more paroxetine immediate release was associated with a higher discontinuation rate compared with escitalopram. ${ }^{7}$ As the National Institute for Health and Care Excellence guidelines indicated, a higher incidence of discontinuation symptoms is observed for paroxetine immediate release than for other selective serotonin reuptake inhibitors (SSRIs). ${ }^{8}$ In order to overcome these drawbacks of paroxetine immediate release, paroxetine controlled release was developed in Japan in 2012 to improve general tolerability, particularly, gastrointestinal tolerability. Although there are no published data which demonstrate that paroxetine controlled release has a lower risk for producing discontinuation effects than paroxetine immediate release, one randomized trial showed that paroxetine controlled release is associated with low rates of early-onset nausea and dropout rates due to adverse events, which were comparable to those of placebo. ${ }^{9}$ However, there are no direct comparisons between escitalopram and paroxetine controlled release in patients with MDD. Therefore, we conducted a 24-week, rater-masked, randomized trial of escitalopram versus paroxetine controlled release in Japanese patients with MDD.

\section{Methods \\ Subjects}

This study was conducted from July 2013 to December 2015 at the Fujita Health University Hospital, Jindai Clinic, Jindai Hospital, Toyota Memorial Hospital, Holy Cross Hospital, and Okehazama Hospital. The trial was registered at the University Hospital Medical Information Network (UMIN) Clinical Trials Registry (UMIN000011191). Patients were diagnosed according to the Diagnostic and Statistical Manual of Mental Disorders, Fourth Edition, Text Revision criteria by the consensus of at least two experienced psychiatrists on the basis of structured interviews conducted using the MiniInternational Neuropsychiatric Interview and a review of all medical records. All subjects met the following inclusion criteria: 1) age 20-70 years; 2) a 17-item Hamilton Rating Scale for Depression (HAMD-17), ${ }^{1}$ with total score at baseline being $\geq 20 ; 3$ ) no neurologic or systemic diseases, including disturbance of hematopoiesis; 4) no history of electroconvulsive therapy within 6 months before enrollment; 5) absence of pregnancy; and 6) no dependence on any addictive substances other than nicotine in the past 5 years before enrollment. All subjects underwent laboratory blood testing and electrocardiography at the time of enrollment, and no patients were excluded because of a medical condition.

The clinical trial was described in detail, and written informed consent was obtained from all participants and their guardians. This study was approved by the six ethics committees of the Fujita Health University Hospital, Jindai Clinic, Jindai Hospital, Toyota Memorial Hospital, Holy Cross Hospital, and Okehazama Hospital.

\section{Procedures}

All patients were randomly assigned to one of the two treatment groups by the central registration office. When the patients satisfied the inclusion and exclusion criteria for this multicenter trial, the randomization procedure was conducted by the authors in contact with a central registration office by telephone. The authors generated an allocation schedule before the start of the study using a random number table. The initial starting dose of escitalopram was 5-10 mg/day, with dose increases when needed in increments of 5-10 mg/day to a maximum of $20 \mathrm{mg} / \mathrm{day}$. The initial starting dose of the paroxetine controlled release was $12.5 \mathrm{mg} /$ day, with a dose increase of 12.5-50 mg/day. The subsequent antidepressant dosage was increased or decreased according to the patient's tolerance and therapeutic response. Lorazepam was used as an anxiolytic, and the hypnotics brotizolam and eszopiclone were permitted during our clinical trial (by prescription and for valid clinical reasons).

\section{Evaluation of psychopathology, tolerability, and safety}

The 21-item HAMD (HAMD-21) ${ }^{1}$ was completed at baseline and at 2, 4, 8, 12, and 24 weeks after starting administration or at discontinuation. Treatment-emergent adverse events were recorded at each time point, including immediately before the study, upon spontaneous complaints and through clinical observation using the Udvalg for Kliniske Undersogelser Side Effect Rating Scale. ${ }^{10}$

The raters who administered the psychometric tests were masked to the treatment group allocation, but the patients and physicians were aware of the treatment that was received. Raters consisted of psychologists who were not involved in any treatment decisions or the evaluation of side effects. Therefore, they were not required to know the pharmacologic treatment. The following measures were taken to maintain the masking of the raters. Electronic data from the trial were password protected, all case report forms were securely stored, and discussions about patients among the research teams were restricted. Moreover, the participants were reminded to avoid open discussion of the treatment assignment with the raters.

The primary outcome measure was the improvement of the HAMD-21 total scores at week 24. The secondary outcomes included: the HAMD-21 scores at 2, 4, 8, and 
12 weeks; the HAMD-17 scores at 2, 4, 8, 12, and 24 weeks; the response rate at $2,4,8,12$, and 24 weeks (clinical response defined as $\geq 50 \%$ reduction in the HAMD-17 or -21 total score from baseline to endpoint); the remission rate at $2,4,8,12$, and 24 weeks (clinical remission defined as the HAMD-17 total score $\leq 6$ or HAMD-21 total score $\leq 7$ ); ${ }^{11}$ the discontinuation rate; and individual adverse events.

\section{Statistical analysis}

Modified intent-to-treat analysis was performed using the last observation carried forward method. Baseline continuous and categorical variables were compared between the treatment groups by an independent $t$-test and a chi-square test, respectively. Paired Student's $t$-tests were used to assess the changes in the HAMD-21 and HAMD-17 total scores from the baseline to endpoint. The comparison between the escitalopram and paroxetine controlled-release treatment groups was made by determining the change in the HAMD-21 (or HAMD-17) total score at the endpoint (2, 4, 8, 12, and 24 weeks) from baseline, based on an analysis of covariance model using the baseline HAMD-21 (or HAMD-17) score as covariates. We also used multiple logistic regression analyses to examine which antidepressant was associated with a higher response rate or remission rate (dependent variable: response rate [HAMD-21 or HAMD-17] or remission rate [HAMD-21 or HAMD-17]; independent variable: treatment group; and covariates: baseline HAMD-21 or HAMD-17 score, respectively). A Kaplan-Meier analysis was used to estimate the time to discontinuation for the two treatment groups, and the results were compared using a log-rank test. We also compared the change in body weight from baseline to endpoint between the escitalopram and paroxetine controlledrelease treatment groups based on an analysis of covariance model using the baseline value as a covariate. Incidences of individual adverse events during the study were compared between the treatment groups using a chi-square test. The final dose of antidepressants was evaluated based on the fluoxetine equivalent. ${ }^{12}$ The statistical power regarding the primary outcome for efficacy was also calculated (alpha $=0.5$, http://www.biostat.ucsf.edu/sampsize.html).

Statistical analyses were performed using Statistical Package for the Social Sciences Statistics for Windows (Version 22.0; Corporation, Armonk, NY, USA) and JMP (JMP 12.2. $1 \mathrm{~J}$; SAS Japan Inc., Tokyo, Japan). A $P$-value of $<0.05$ was considered statistically significant for all tests.

\section{Results}

The demographics and other characteristics of the patients are presented in Table 1. A total of 88 patients were recruited, all of whom were diagnosed with MDD at enrollment. Of these, $77.3 \%$ were first-episode patients and $61.4 \%$ were male, and the mean age was $40.8 \pm 13.4$ years. There were no significant differences in most variables between the treatment groups (Table 1).

Table I Baseline characteristics of the patients

\begin{tabular}{|c|c|c|c|c|}
\hline Variable & $\begin{array}{l}\text { Escitalopram } \\
(n=43)\end{array}$ & $\begin{array}{l}\text { Paroxetine controlled } \\
\text { release }(n=45)\end{array}$ & $\begin{array}{l}\text { Group difference } \\
\left(\chi^{2} \text { test or } t \text {-test }\right)\end{array}$ & $P$-value \\
\hline Male (\%) & $25(58.1)$ & $29(64.4)$ & $\chi^{2}=0.369$ & 0.544 \\
\hline Age, years (mean $\pm S D$ ) & $38.9 \pm 12.4$ & $42.5 \pm 14.2$ & $t=-1.28$ & 0.205 \\
\hline First episode (\%) & $35(81.4)$ & $33(73.3)$ & $\chi^{2}=0.819$ & 0.366 \\
\hline Educational history, years (mean $\pm S D$ ) & $14.0 \pm 1.88$ & $13.6 \pm 2.22$ & $t=-0.752$ & 0.454 \\
\hline Hospitalization (\%) & $0(0.00)$ & I $(2.22)$ & $\chi^{2}=1.35$ & 0.245 \\
\hline Study center (FHU hospital, \%) & $19(44.2)$ & $26(57.8)$ & $\chi^{2}=1.63$ & 0.202 \\
\hline Married (\%) & $25(58.1)$ & $19(42.2)$ & $\chi^{2}=2.24$ & 0.135 \\
\hline Unemployed (\%) & $8(18.6)$ & $12(26.7)$ & $\chi^{2}=0.819$ & 0.366 \\
\hline Current smoker (\%) & $9(20.9)$ & $9(20.0)$ & $\chi^{2}=0.012$ & 0.914 \\
\hline $\begin{array}{l}\text { Hamilton Depression Rating Scale-17 } \\
\text { total scores (mean } \pm \text { SD) }\end{array}$ & $23.7 \pm 3.58$ & $23.2 \pm 3.78$ & $t=-0.605$ & 0.547 \\
\hline $\begin{array}{l}\text { Hamilton Depression Rating Scale-2I } \\
\text { total scores (mean } \pm \text { SD) }\end{array}$ & $25.8 \pm 4.24$ & $25.1 \pm 4.7 I$ & $t=-0.710$ & 0.480 \\
\hline Comorbid psychiatric illness (\%)* & $6(14.0)$ & $6(13.3)$ & $\chi^{2}=0.007$ & 0.933 \\
\hline Body weight, kg (mean \pm SD) & $65.0 \pm 13.9$ & $65.7 \pm 12.3$ & $t=0.240$ & 0.811 \\
\hline Use of antidepressant (\%) & $0(0.00)$ & $0(0.00)$ & Not applicable & \\
\hline Use of hypnotic (\%) & $6(14.0)$ & $6(13.3)$ & $\chi^{2}=0.007$ & 0.933 \\
\hline Use of anxiolytic (\%) & $0(0.00)$ & I (2.22) & $\chi^{2}=1.35$ & 0.245 \\
\hline
\end{tabular}

Note: *Generalized anxiety disorder and/or panic disorder.

Abbreviations: FHU, Fujita Health University; SD, standard deviation. 
Table 2 Discontinuation rate

\begin{tabular}{lll}
\hline Number of patients & Escitalopram & $\begin{array}{l}\text { Paroxetine } \\
\text { controlled release }\end{array}$ \\
\hline Randomized & 43 & 45 \\
Completed the trial (\%) & $19(44.2)$ & $18(40.0)$ \\
Discontinued the trial (\%) & $24(55.8)$ & $27(60.0)$ \\
The reasons of discontinuation & \\
Inefficacy (\%) & $9(20.9)$ & $8(17.8)$ \\
Adverse events (\%) & $8(18.6)$ & $11(24.4)$ \\
Other reasons (\%) & $7(16.2)$ & $8(17.8)$ \\
\hline
\end{tabular}

The discontinuation rate was $58.0 \%$ (escitalopram, $55.8 \%$ and paroxetine controlled release, $60.0 \%$; Table $2)$. There was no significant difference in the mean times to discontinuation between the escitalopram and paroxetine controlled-release treatment groups (13.4 \pm 10.1 vs $13.2 \pm 9.92$ weeks; $\left.\chi_{(1)}^{2}=0.0836, P=0.773\right)$. All 88 patients were included in the efficacy and safety analyses. The mean escitalopram and paroxetine controlled-release doses at endpoint were $13.6 \pm 5.81 \mathrm{mg} /$ day (fluoxetine equivalent, $30.2 \pm 12.9 \mathrm{mg} /$ day) and $24.8 \pm 13.3 \mathrm{mg} /$ day (fluoxetine equivalent, $29.2 \pm 15.7 \mathrm{mg} /$ day), respectively $\left(t_{(1)}=0.0836, P=0.773\right)$.

Both escitalopram and paroxetine controlled-release treatments were associated with significant improvements in the HAMD-21 and HAMD-17 total scores at 2, 4, 8, 12, and 24 weeks (Table S1). However, there were no significant differences in the magnitude of the HAMD-21, and HAMD-17 total score decreases at any time between the escitalopram and paroxetine controlled-release treatment groups (Table 3). The statistical power with respect to the primary outcome was $43 \%$. Also, there were no significant differences in the response rate at any time point between the treatment groups (Figures 1 and 2).

No patients had serious adverse events such as death, suicide attempt, and serotonin syndrome, and there were no significant differences in the incidence of individual adverse events between the groups (Table 4). Moreover, there were no significant differences in body weight at the endpoint between the treatment groups (Table 4).

\section{Discussion}

This is the first randomized trial involving a comparison between escitalopram and paroxetine controlled release in Japanese patients with MDD. There were no significant differences in the efficacy and safety outcomes between the escitalopram and paroxetine controlled-release treatment groups in this study. However, our study found that discontinuation rates due to adverse events in both treatment

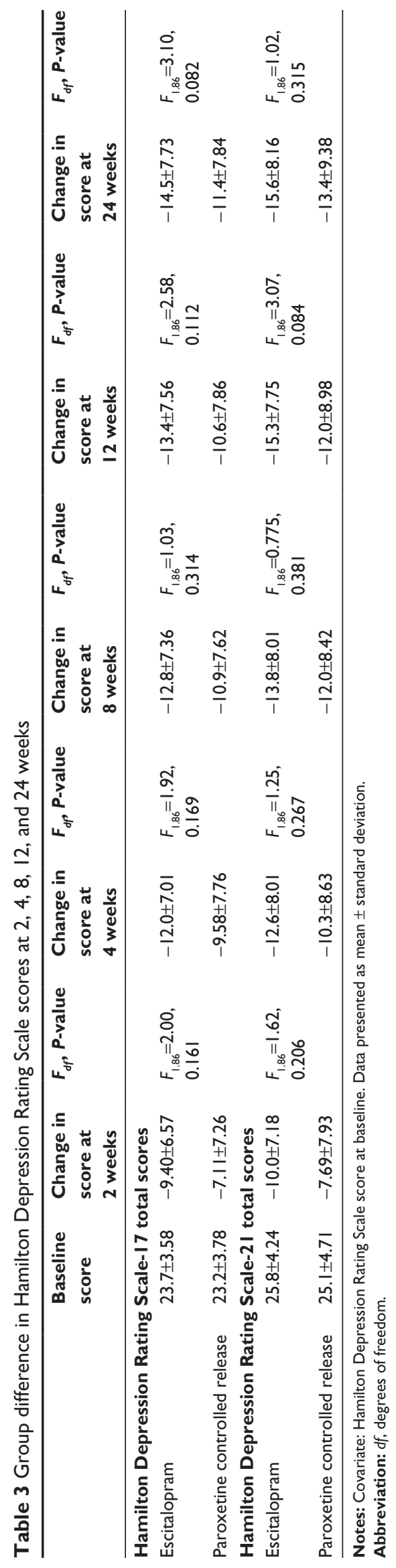


HAMD-17

\section{0}

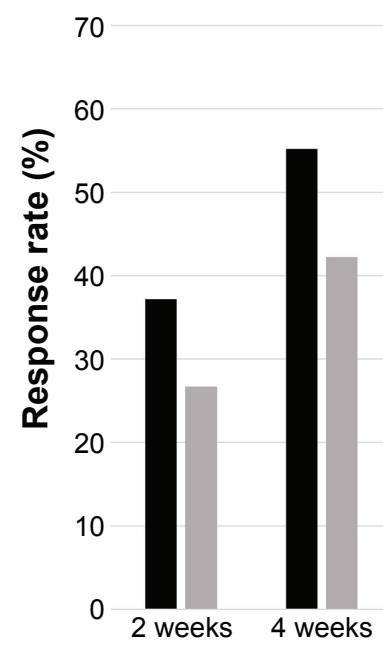

Figure I Response rate.

Abbreviations: CR, controlled release; HAMD, Hamilton Depression Rating Scale.
HAMD-21

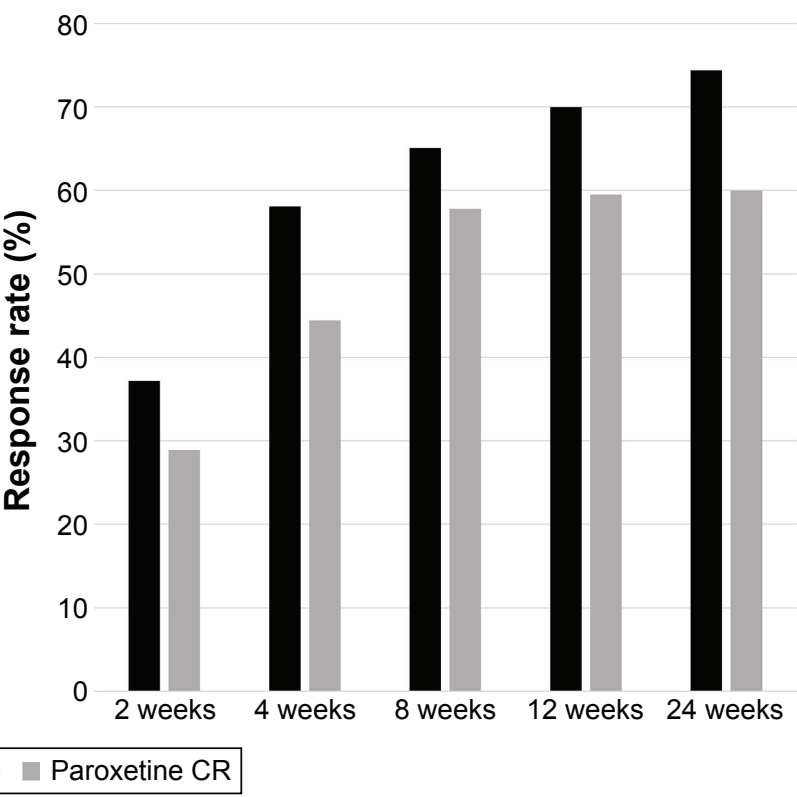

groups were relatively high compared with other studies on SSRIs. ${ }^{13}$ In a recent network meta-analysis, escitalopram emerged as the most efficacious agent among the SSRIs and was the best tolerated of the new-generation antidepressants (eg, agomelatine, duloxetine, escitalopram, fluvoxamine, fluoxetine, mirtazapine, paroxetine, sertraline, trazodone, and venlafaxine) that were analyzed. ${ }^{13}$ We did not find significant differences in any efficacy outcome between the escitalopram and paroxetine controlled-release treatment groups in this study. The statistical power with respect to the primary outcome was $43 \%$. Therefore, our results might be a statistical error due to an insufficient sample size.
HAMD-17

60

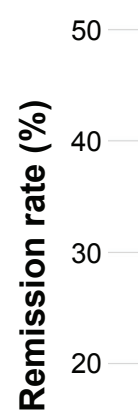

10

0

2 weeks

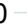

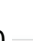

20

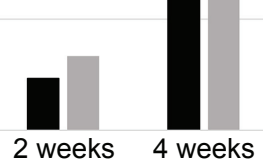

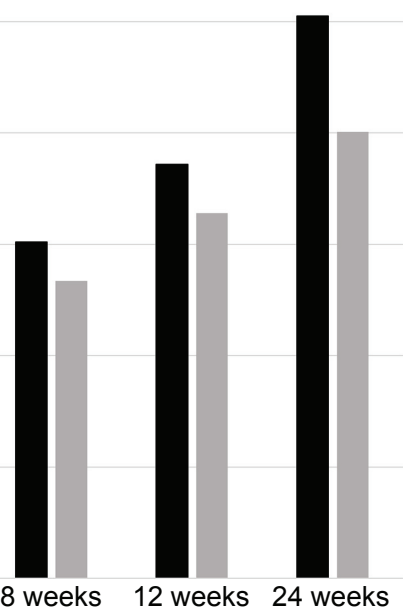

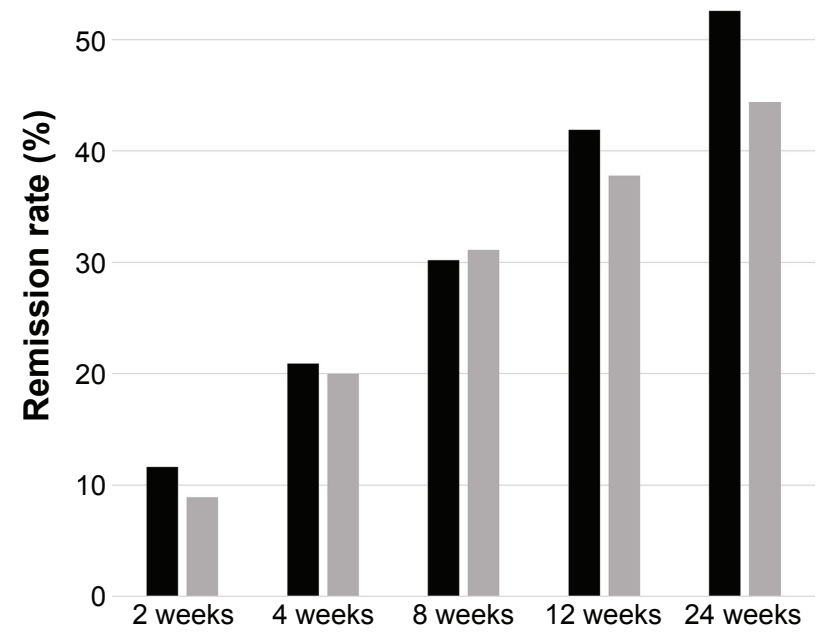

Escitalopram Paroxetine CR

Figure 2 Remission rate.

Abbreviations: CR, controlled release; HAMD, Hamilton Depression Rating Scale. 
Table 4 Adverse events observed in the study (incidence $\geq 5 \%$ )

\begin{tabular}{|c|c|c|c|}
\hline Individual adverse events & $\begin{array}{l}\text { Escitalopram } \\
(n=43)\end{array}$ & $\begin{array}{l}\text { Paroxetine controlled } \\
\text { release }(n=45)\end{array}$ & $\begin{array}{l}\text { Group difference }\left(\chi^{2} \text { test or }\right. \\
\text { analysis of covariance and } P \text {-value }\end{array}$ \\
\hline At least one adverse event (\%) & $26(60.5)$ & $29(64.4)$ & $\chi^{2}=0.149$ and 0.700 \\
\hline Headache $(\%)$ & $5(11.6)$ & $2(4.44)$ & $\chi^{2}=1.59$ and 0.207 \\
\hline Somnolence (\%) & $12(27.9)$ & $8(17.8)$ & $\chi^{2}=1.29$ and 0.256 \\
\hline Dizziness (\%) & $6(14.0)$ & $4(8.89)$ & $\chi^{2}=0.562$ and 0.453 \\
\hline Anxiety (\%) & $9(20.9)$ & $4(8.89)$ & $\chi^{2}=2.58$ and 0.108 \\
\hline Insomnia (\%) & $6(14.0)$ & $5(11.1)$ & $\chi^{2}=0.162$ and 0.687 \\
\hline Decreased salivation (\%) & $3(6.98)$ & $5(11.1)$ & $\chi^{2}=0.460$ and 0.498 \\
\hline Nausea/vomiting (\%) & II (25.6) & $12(26.7)$ & $\chi^{2}=0.013$ and 0.908 \\
\hline Diarrhea (\%) & $9(20.9)$ & $6(13.3)$ & $\chi^{2}=0.901$ and 0.342 \\
\hline Constipation (\%) & $6(14.0)$ & $3(6.67)$ & $\chi^{2}=1.29$ and 0.256 \\
\hline Loss of sexual desire (\%) & $2(4.65)$ & $3(6.67)$ & $\chi^{2}=0.168$ and 0.682 \\
\hline Use of hypnotic (\%) & $6(14.0)$ & $6(13.3)$ & $\chi^{2}=0.007$ and 0.933 \\
\hline Use of anxiolytic (\%) & $0(0.00)$ & I (2.22) & $\chi^{2}=1.35$ and 0.245 \\
\hline Weight gain (\%) & $\mathrm{I}(2.33)$ & $3(6.67)$ & $\chi^{2}=1.00$ and 0.317 \\
\hline Mean weight change $(\mathrm{kg})^{*}$ & $0.853 \pm 3.05$ & $0.502 \pm 2.11$ & $F_{1.86}=0.47 \mathrm{I}$ and 0.494 \\
\hline
\end{tabular}

Notes: *Covariate: body weight at baseline. Data presented as mean \pm standard deviation.

\section{Limitations}

One of the primary limitations of this study is that this study was not double blinded. The physicians responsible for assessing the adverse events were aware of which treatment was received by which patient. Although the raters were masked to the nature of the antidepressant treatment and were not involved in any side effect ratings or management decisions, it is possible that they were inadvertently informed of the group allocation by the patients. The second limitation is that this study did not include a placebo arm. The third limitation is the small sample size, particularly in the subgroup analysis (the statistical power with respect to the primary outcome was $43 \%$ ). Therefore, we did not perform an HAMD item analysis. The fourth limitation is that we did not correct for multiple comparisons (eg, using Bonferroni's correction), because the application of a more stringent alpha level for secondary outcomes would have been too conservative in this small sample. ${ }^{14,15}$ The fifth one is that we did not count the number of patients who were screened for entry in the study. Finally, the intent-to-treat analysis using the last observation carried forward method may have influenced the results. The discontinuation rate was high, and thus, this method may yield a biased estimate of the treatment effect and underestimate the variability of the results. ${ }^{16}$ It is not clearly justified why this analysis, in particular, is appropriate and valid. However, if we had selected a complete analysis, our sample size would have been too small.

\section{Conclusion}

In conclusion, our findings suggest that although escitalopram and paroxetine controlled release are effective in patients with
MDD, discontinuation rates due to adverse events in both treatment groups were relatively high. However, because our study might be underpowered to detect significant differences in efficacy and safety outcomes between the escitalopram and paroxetine controlled-release treatment groups, further study using a larger sample size is required.

\section{Acknowledgments}

We thank the following colleagues who shared their data with us: Ms M Yamamoto, Ms R Nishioka, Ms Y Matsumoto, Ms M Tani, Ms S Isogai, Ms M Niwa, Ms M Miyako, Ms E Tsutajima, Ms N Soma, Ms A Hayashi, Mr W Shirota, Mr U Sato, Mr S Tsuboi, and Ms K Torii.

This study was supported by Grant-in-Aid for Young Scientists (B) (25861033) and Fujita Health University School of Medicine research grant.

\section{Disclosure}

The authors declare that the research was conducted in the absence of any commercial or financial relationships that could be construed as a potential conflict of interest.

Dr Kishi has received speaker's honoraria from Abbvie, Astellas, Daiichi Sankyo, Dainippon Sumitomo, Eisai, Eli Lilly, GlaxoSmithKline, Janssen, Yoshitomi, Otsuka, Meiji, Mochida, Shionogi, Tanabe-Mitsubishi, Tsumura, Novartis, and Pfizer and has a Fujita Health University School of Medicine research grant and Grant-in-Aid for Young Scientists (B). Dr Matsuda has received speaker's honoraria from Dainippon Sumitomo, Eli Lilly, Otsuka, and Pfizer. Dr Matsunaga has received speaker's honoraria from Eisai, Janssen, Novartis, Daiichi Sankyo, Ono, Eli 
Lilly, Takeda, and Otsuka and had a Fujita Health University School of Medicine research grant and Grant-in-Aid for Young Scientists (B). Dr Moriwaki has received speaker's honoraria from Eli Lilly, Yoshitomi, and Novartis. Dr Otake has received speaker's honoraria from Dainippon Sumitomo, Eli Lilly, and Otsuka. Dr Akamatsu has received speaker's honoraria from Dainippon Sumitomo, Eli Lilly, and Otsuka. Dr Funahashi has received speaker's honoraria from Astellas, Dainippon Sumitomo, Eli Lilly, GlaxoSmithKline, Janssen, Yoshitomi, Otsuka, Meiji, and Pfizer. Dr Tabuse has received speaker's honoraria from Astellas, Dainippon Sumitomo, Eli Lilly, GlaxoSmithKline, Janssen, Yoshitomi, Otsuka, Meiji, and Pfizer. Dr Fujita has received speaker's honoraria from Eli Lilly, Mochida, GlaxoSmithKline, Janssen, Yoshitomi, and Otsuka. Dr Iwata has received speaker's honoraria from Astellas, Dainippon Sumitomo, Eli Lilly, GlaxoSmithKline, Janssen, Yoshitomi, Otsuka, Meiji, Shionogi, Novartis, and Pfizer. The authors report no other conflicts of interest in this work.

\section{References}

1. Hamilton M. A rating scale for depression. J Neurol Neurosurg Psychiatry. 1960;23:56-62.

2. Cipriani A, Furukawa TA, Salanti G, et al. Comparative efficacy and acceptability of 12 new-generation antidepressants: a multiple-treatments meta-analysis. Lancet. 2009;373(9665):746-758.

3. Ramsberg J, Asseburg C, Henriksson M. Effectiveness and costeffectiveness of antidepressants in primary care: a multiple treatment comparison meta-analysis and cost-effectiveness model. PLoS One. 2012; 7(8):e42003.

4. Ai Report 2011 -chikenyakunenpou- [The sales amounts of antidepressants in Japan]. CIMA Sci J. 2011.
5. Boulenger JP, Huusom AK, Florea I, Baekdal T, Sarchiapone M A comparative study of the efficacy of long-term treatment with escitalopram and paroxetine in severely depressed patients. Curr Med Res Opin. 2006;22(7):1331-1341.

6. Kasper S, Baldwin DS, Larsson Lonn S, Boulenger JP. Superiority of escitalopram to paroxetine in the treatment of depression. Eur Neuropsychopharmacol. 2009;19(4):229-237.

7. Baldwin DS, Cooper JA, Huusom AK, Hindmarch I. A double-blind, randomized, parallel-group, flexible-dose study to evaluate the tolerability, efficacy and effects of treatment discontinuation with escitalopram and paroxetine in patients with major depressive disorder. Int Clin Psychopharmacol. 2006;21(3):159-169.

8. NICE. Depression in Adults: Recognition and Management [Last updated: April 2016]. National Institute for Health and Care Excellence; 2009.

9. Golden RN, Nemeroff CB, McSorley P, Pitts CD, Dube EM. Efficacy and tolerability of controlled-release and immediate-release paroxetine in the treatment of depression. J Clin Psychiatry. 2002;63(7):577-584.

10. Jordan S, Knight J, Pointon D. Monitoring adverse drug reactions: scales, profiles, and checklists. Int Nurs Rev. 2004;51(4):208-221.

11. Riedel M, Moller HJ, Obermeier M, et al. Response and remission criteria in major depression - a validation of current practice. J Psychiatr Res. 2010;44(15):1063-1068.

12. Hayasaka Y, Purgato M, Magni LR, et al. Dose equivalents of antidepressants: evidence-based recommendations from randomized controlled trials. J Affect Disord. 2015;180:179-184.

13. Khoo AL, Zhou HJ, Teng M, et al. Network meta-analysis and costeffectiveness analysis of new generation antidepressants. CNS Drugs. 2015;29(8):695-712.

14. Perneger TV. What's wrong with Bonferroni adjustments. BMJ. 1998; 316(7139):1236-1238.

15. Sankoh AJ, Huque MF, Dubey SD. Some comments on frequently used multiple endpoint adjustment methods in clinical trials. Stat Med. 1997;16(22):2529-2542.

16. Mallinckrodt CH, Kaiser CJ, Watkin JG, Molenberghs G, Carroll RJ. The effect of correlation structure on treatment contrasts estimated from incomplete clinical trial data with likelihood-based repeated measures compared with last observation carried forward ANOVA. Clin Trials. 2004;1(6):477-489. 


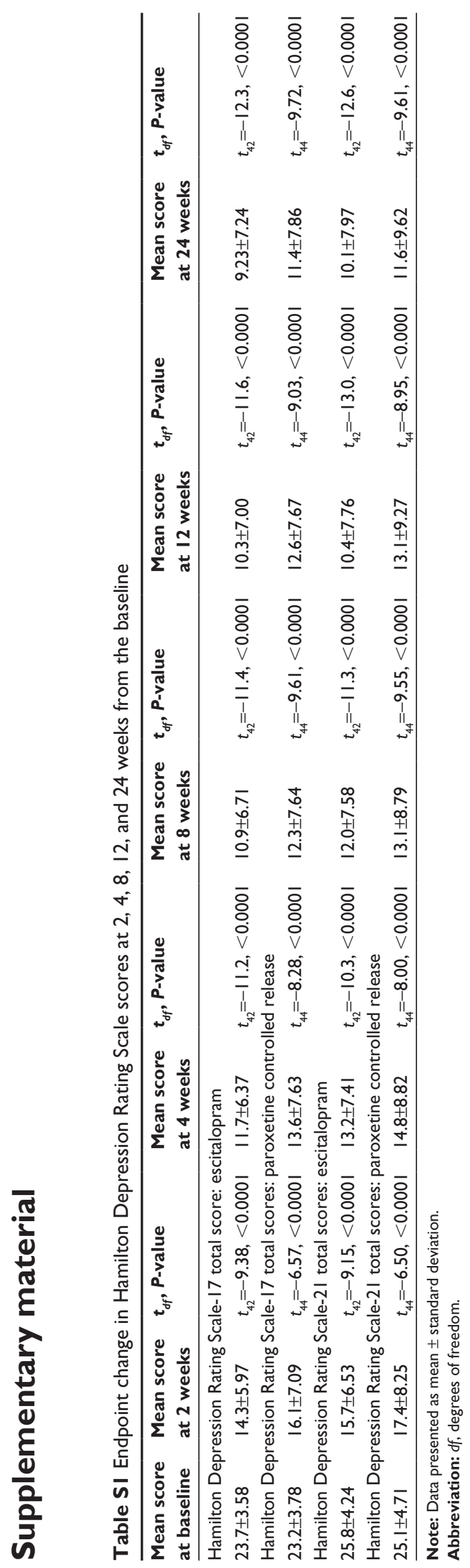




\section{Publish your work in this journal}

Neuropsychiatric Disease and Treatment is an international, peerreviewed journal of clinical therapeutics and pharmacology focusing on concise rapid reporting of clinical or pre-clinical studies on a range of neuropsychiatric and neurological disorders. This journal is indexed on PubMed Central, the 'PsycINFO' database and CAS, and is the official journal of The International Neuropsychiatric Association (INA). The manuscript management system is completely online and includes a very quick and fair peer-review system, which is all easy to use. Visit http://www.dovepress.com/testimonials.php to read real quotes from published authors.

Submit your manuscript here: http://www.dovepress.com/neuropsychiatric-disease-and-treatment-journal 